\title{
Characteristics of Cutaneous Lymphomas in Korea According to the New WHO-EORTC Classification: Report of a Nationwide Study
}

Jae Ho Han • Young-Hyeh Ko ${ }^{1}$ Yun Kyung Kang ${ }^{2}$. Wan-Seop Kim ${ }^{3}$ Yoon Jung $\mathrm{Kim}^{4}$. Insun $\mathrm{Kim}^{5}$ Hyun-Jung Kim ${ }^{6}$. Soo Kee Min ${ }^{7}$ Chan-Kum Park ${ }^{8}$. Chan-Sik Park ${ }^{9}$ Bong-Kyung Shin ${ }^{10} \cdot$ Woo Ick Yang ${ }^{11}$ Young-Ha Oh ${ }^{12} \cdot$ Jong Sil Lee ${ }^{13}$ Juhie Lee ${ }^{14} \cdot$ Tae Hui Lee ${ }^{15}$ Hyekyung Lee ${ }^{16} \cdot$ Ho Jung Lee ${ }^{17}$ Yoon Kyung Jeon ${ }^{18} \cdot$ Hee Jeong Cha ${ }^{19}$ Yoo-Duk $\mathrm{Choi}^{20}$. Chul Woo Kim ${ }^{18}$ Hematopathology Study Group of the Korean Society of Pathologists

Department of Pathology, Ajou University School of Medicine, Suwon; 'Samsung Medical Center, Sungkyunkwan University School of Medicine, Seoul; 2Inje University Seoul Paik Hospital, Seoul; ${ }^{3}$ Konkuk University School of Medicine, Seoul; ${ }^{4}$ Veterans Health Service Medical Center, Seoul; ${ }^{5}$ Korea University College of Medicine, Seoul; ${ }^{6}$ Inje University Sanggye Paik Hospital, Seoul; ${ }^{7}$ Hallym University Sacred Heart Hospital, Anyang; ${ }^{8}$ Hanyang University College of Medicine, Seoul; ${ }^{9}$ Asan Medical Center, Seoul; ${ }^{10}$ Korea University Guro Hospital, Seoul;; ${ }^{11}$ Yonsei University College of Medicine, Seoul; ${ }^{12}$ Hanyang University Guri Hospital, Guri; ${ }^{13}$ Gyeongsang National University School of Medicine, Jinju; ${ }^{14}$ Kyung Hee University School of Medicine, Seoul; ${ }^{15}$ Dankook University College of Medicine, Cheonan; ${ }^{16}$ Eulji University Hospital, Daejeon; ${ }^{17}$ Eulji General Hospital, Seoul; ${ }^{18}$ Seoul National University, Seoul; ${ }^{19}$ Ulsan University College of Medicine, Ulsan; ${ }^{20}$ Chonnam National University Medical School, Gwangju, Korea

Received: January 16, 2014

Revised: February 11, 2014

Accepted: February 14, 2014

Corresponding Author

Chul Woo Kim, M.D.

Department of Pathology, Seoul National University, 101 Daehak-ro, Jongno-gu, Seoul 110-744, Korea Tel: +82-2-740-8267

Fax: $+82-2-743-5530$

E-mail: cwkim@snu.ac.kr
Background: Previously, cutaneous lymphomas were classified according to either the European Organization for the Research and Treatment of Cancer (EORTC) or the World Health Organization $(\mathrm{WHO})$ classification paradigms. The aim of this study was to determine the relative frequency of Korean cutaneous lymphoma according to the new WHO-EORTC classification system. Methods: A total of 517 patients were recruited during a recent 5 year-period (2006-2010) from 21 institutes and classified according to the WHO-EORTC criteria. Results: The patients included 298 males and 219 females, and the mean age at diagnosis was 49 years. The lesions preferentially affected the trunk area (40.2\%). The most frequent subtypes in order of decreasing prevalence were mycosis fungoides (22.2\%), peripheral T-cell lymphoma (17.2\%), CD30+ T-cell lymphoproliferative disorder (13.7\%), and extranodal natural killer/T (NK/T) cell lymphoma, nasal type (12.0\%). Diffuse large B-cell lymphoma accounted for $11.2 \%$ of cases, half of which were secondary cutaneous involvement; other types of B-cell lymphoma accounted for less than $1 \%$ of cases. Conclusions: In comparison with data from Western countries, this study revealed relatively lower rates of mycosis fungoides and B-cell lymphoma in Korean patients, as well as higher rates of subcutaneous panniculitis-like T-cell lymphoma and NK/T cell lymphoma. 
Cutaneous lymphoma represents $4 \%$ of the total number of malignant lymphoma cases in Korea. ${ }^{1}$ Lymphoma may involve the skin as the primary and only site of involvement or as a secondary site of disease. Some lymphomas are present only in the skin, where they resemble their nodal counterparts histologically but nevertheless represent distinct entities. ${ }^{2}$ Currently, cutaneous lymphomas are classified according to either the European Organization for the Research and Treatment of Cancer (EORTC) or the World Health Organization (WHO) classification systems. Following a consensus meetings of the two organizations, a joint WHO-EORTC classification was developed. ${ }^{2,3}$ Compared with $2001 \mathrm{WHO}$ classification system, primary cutaneous aggressive epidermotropic CD8-positive cytotoxic Tcell lymphoma, primary cutaneous $\gamma \delta$ T-cell lymphoma, and primary cutaneous small/medium CD4-positive T-cell lymphoma are included as provisional entities, and variants of diffuse large B-cell lymphoma (DLBCL) are also described. In addition, the 4th edition of the WHO classification system includes blastic plasmacytoid dendritic cell (BPDC) neoplasms previously categorized as blastic natural killer (NK)-cell lymphoma. ${ }^{2,4}$

To date, several large scale studies on cutaneous lymphoma have been performed in Western countries. ${ }^{3,5,6}$ However, literature on Korean cutaneous lymphomas is limited because of the rarity of cases and thus the inability to study large numbers of patients. In the present study, we analyzed the incidence of cutaneous lymphoma in Korean patients according to the new WHO-EORTC classification system.

\section{MATERIALS AND METHODS}

A total of 517 cases of cutaneous lymphoma diagnosed at 21

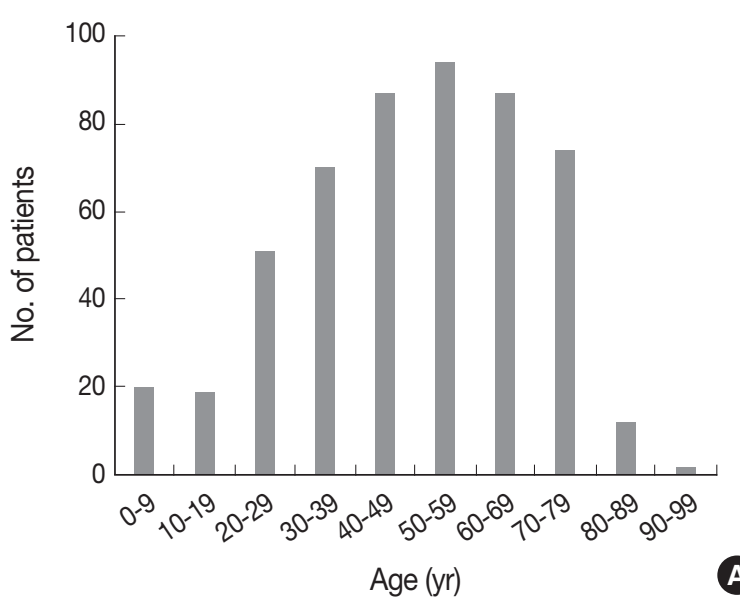

institutes in Korea between January 2006 and December 2010 were enrolled in our study. The cases were reviewed by hematopathologists and re-classified according to the new WHO-EORTC classification. ${ }^{2-4}$ Clinical data including age, sex, site of involvement, and primary or secondary disease were obtained. The histologic and clinical results were compared with those of previous Western studies.

\section{RESULTS}

Patients were aged between 1 and 91 years (mean, 49 years). Although any age group may be involved, cutaneous lymphoma was most common in patients in their fifth, sixth, and seventh decades, with a peak incidence at approximately 60 years of age (Fig. 1A). Patients included 298 males (57.6\%) and 219 females (42.4\%) (male:female ratio, 1.4:1). Lesions could be present at any site, but the trunk was the most common anatomic site (40.2\%) (Fig. 1B).

The overall incidence of cutaneous lymphoma according to the WHO-EORTC classification is summarized in Table 1. Among a total of 517 cases of cutaneous lymphoma, primary and secondary cutaneous lymphomas accounted for $73.1 \%$ and $26.1 \%$, respectively, while only $0.8 \%$ of cases were undetermined. Among all 517 patients, the relative frequencies of mature T-cell lymphoma, mature B-cell lymphoma, immature hematopoeitic malignancy and Hodgkin's lymphoma were 74.3\%, 18.0\%, 5.8\%, and $0.2 \%$, respectively. Further, for the 378 cases of primary cutaneous lymphomas, the proportion of T-cell lymphoma (82.3\%) increased when compared with the proportion of total cases.

The most frequent subtypes, in decreasing order of prevalence, were mycosis fungoides (MF) including its variants (22.2\%), pe-

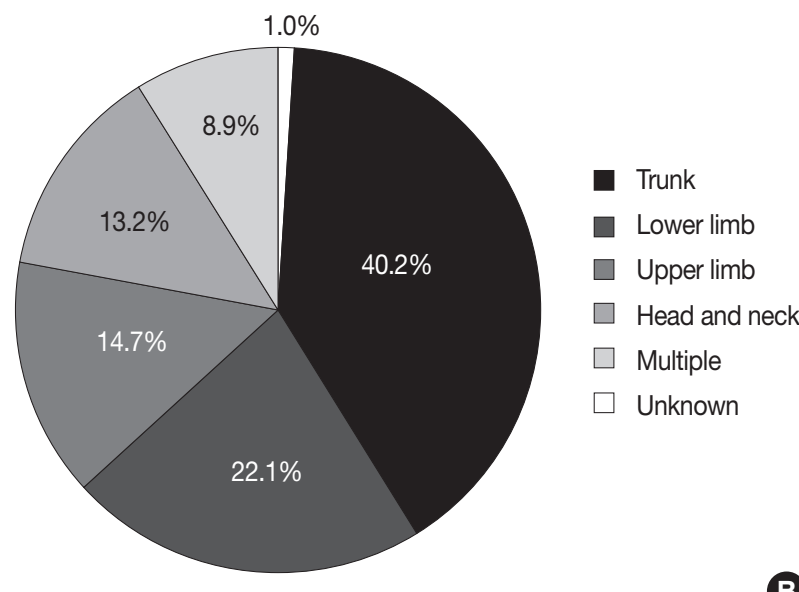

Fig. 1. Age (A) and site (B) distribution of cutaneous lymphoma. 
Table 1. Incidence of cutaneous lymphoma according to WHO-EORTC classification in Korea between 2006 and 2010

\begin{tabular}{|c|c|c|c|}
\hline & Total & Primary & Secondary \\
\hline \multicolumn{4}{|l|}{ Mature T-cell lymphoma } \\
\hline Mycosis fungoides & $110(21.3)$ & $110(29.1)$ & - \\
\hline Pagetoid reticulosis & $1(0.2)$ & $1(0.3)$ & - \\
\hline Follicular, syringotropic, granulomatous variants & $3(0.6)$ & $3(0.8)$ & - \\
\hline Granulomatous slack skin & $1(0.2)$ & $1(0.3)$ & - \\
\hline Sezary syndrome & $1(0.2)$ & $1(0.3)$ & - \\
\hline CD30+ T-cell lymphoproliferative disorders & $3(0.6)$ & $1(0.3)$ & $2(1.5)$ \\
\hline Lymphomatoid papulosis & $32(6.2)$ & $32(8.5)$ & - \\
\hline Anaplastic large cell lymphoma & $36(7.0)$ & $31(8.2)$ & $5(3.7)$ \\
\hline Subcutaneous panniculitis-like T-cell lymphoma & $32(6.2)$ & $31(8.2)$ & $1(0.8)$ \\
\hline Peripheral T-cell lymphoma, unspecified & $71(13.7)$ & $51(13.5)$ & $19(14.2)$ \\
\hline Aggressive epidermotropic CD8-positive cytotoxic T-cell lymphoma & $4(0.8)$ & $4(1.1)$ & - \\
\hline Gamma/delta-positive T-cell lymphoma & $9(1.7)$ & $8(2.1)$ & $1(0.8)$ \\
\hline Small/medium CD4+ T-cell lymphoma & $5(1.0)$ & $5(1.3)$ & - \\
\hline Extranodal NKTT cell lymphoma & $58(11.2)$ & $27(7.1)$ & $30(22.4)$ \\
\hline Hydroa vacciniforme-like lymphoma & $4(0.8)$ & $4(1.1)$ & - \\
\hline Adult T-cell leukemia/lymphoma & $1(0.2)$ & - & - \\
\hline Angioimmunoblastic T-cell lymphoma & $13(2.5)$ & $1(0.3)$ & $12(9.0)$ \\
\hline \multicolumn{4}{|l|}{ Mature B-cell lymphoma } \\
\hline Marginal zone B-cell lymphoma & $23(4.5)$ & $19(5.0)$ & $4(3.0)$ \\
\hline Follicle centre lymphoma & $4(0.8)$ & $4(1.1)$ & - \\
\hline Diffuse large B-cell lymphoma & $43(8.3)$ & $17(4.5)$ & $26(19.4)$ \\
\hline Diffuse large B-cell lylmphoma, leg type & $9(1.7)$ & $6(1.6)$ & $3(2.2)$ \\
\hline Diffuse large B-cell lymphoma, other & $5(1.0)$ & - & $4(3.0)$ \\
\hline Plasmablastic lymphoma & $1(0.2)$ & - & $1(0.8)$ \\
\hline Intravascular large B-cell lymphoma & $4(0.8)$ & $2(0.5)$ & $2(1.5)$ \\
\hline Lymphomatoid granulomatosis & $1(0.2)$ & $1(0.3)$ & - \\
\hline Chronic lymphocytic leukemia & $1(0.2)$ & - & $1(0.8)$ \\
\hline Burkitt lymphoma & $2(0.4)$ & - & $2(1.5)$ \\
\hline \multicolumn{4}{|l|}{ Immature hematopoietic malignancies } \\
\hline Blastic plasmacytoid dendritic cell neoplasm & $11(2.1)$ & $10(2.7)$ & $1(0.8)$ \\
\hline T-lymphoblastic lymphoma/leukemia & $9(1.7)$ & $2(0.5)$ & $7(5.2)$ \\
\hline B-lymphoblastic lymphoma/leukemia & $10(1.9)$ & $3(0.8)$ & $7(5.2)$ \\
\hline Hodgkin's lymphoma & $1(0.2)$ & - & $1(0.8)$ \\
\hline Others & $9(1.7)$ & $3(0.8)$ & $6(4.5)$ \\
\hline
\end{tabular}

Values are presented as number (\%).

WHO, World Health Organization; EORTC, European Organization for the Research and Treatment of Cancer; NK, natural killer.

ripheral T-cell lymphoma (PTCL) including subtype (17.2\%), CD30+ T-cell lymphoproliferative disorder (CD30 + LPD) (13.7\%), and extranodal NK/T cell lymphoma, nasal type (12.0\%), respectively (Fig. 2A). MF variants including pagetoid reticulosis or folliculotropic were rarely described. Of the CD $30+$ LPD, there were 32 (6.2\%) cases of lymphomatoid papulosis (LyP) and $36(7.0 \%)$ cases of anaplastic large cell lymphoma (ALCL). Three cases could not be subclassified, and ALCL included five systemic cases. Half of the NK/T cell lymphoma cases were secondary lymphoma. Other cutaneous T-cell lymphoma included subcutaneous panniculitis-like T-cell lymphoma (SPTCL) (6.2\%), angioimmunoblastic T-cell lymphoma (AITL) (2.5\%), and T lymphoblastic lymphoma (1.7\%). Adult T-cell leukemia/lymphoma was rarely observed $(0.2 \%)$. With respect to primary cutaneous lymphoma, the proportion of MF, CD30+ LPD and SPTCL was increased (Fig. 2B). Among B-cell lymphomas, the DLBCL was the most common lymphoma and accounted for $11.2 \%$ of cases, although half were secondary cutaneous involvement. Cutaneous marginal zone B-cell lymphoma was the second most common B-cell lymphoma (4.5\%), and other types of B-cell lymphoma accounted for less than $1 \%$ of cases. Immature hematopoietic malignancies included BPDC neoplasm (11 cases, 2.1\%), Tlymphoblastic leukemia/lymphoma (9 cases, 1.7\%) and B-lymphoblastic leukemia/lymphoma (10 cases, $1.9 \%$ ).

The DLBCL and PTCL subtypes were rarely observed. Of the 68 primary cutaneous peripheral T-cell lymphomas, 4 cases were primary cutaneous aggressive epidermotropic $\mathrm{CD} 8$-positive cytotoxic T-cell lymphoma, 8 cases were gamma/delta-positive T- 
Total (primary+secondary, 517 cases)

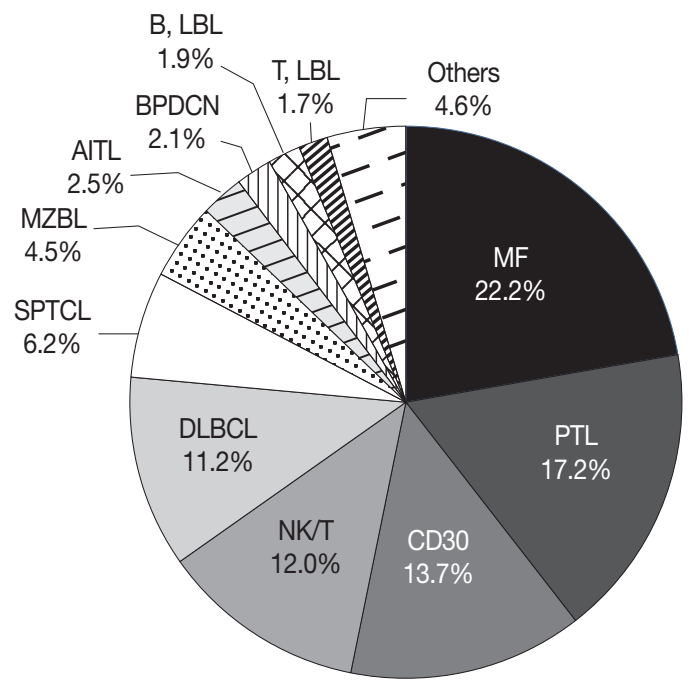

Primary (378 cases)

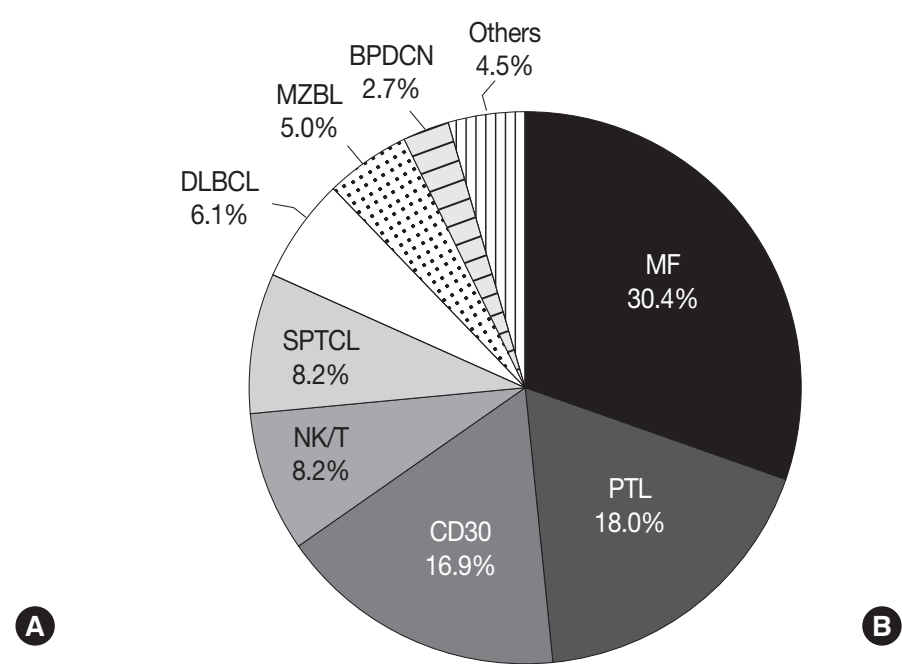

Fig. 2. (A, B) Relative frequency of cutaneous lymphoma according to subtype. MF, mycosis fungoides; PTL, peripheral T-cell lymphoma; CD30, CD30+ T-cell lymphoproliferative disorder; NK/T, NK/T cell lymphoma, nasal type; DLBCL, diffuse large B-cell lymphoma; SPTCL, subcutaneous panniculitis-like T-cell lymphoma; MZBL, marginal zone B-cell lymphoma; AITL, angioimmunoblastic T-cell lymphoma; BPDCN, blastic plasmacytoid dendritic cell neoplasm; LBL, lymphoblastic lymphoma.

Table 2. Cutaneous lymphomas by histologic type, sex, and age

\begin{tabular}{lcrrrrr}
\hline & \multirow{2}{*}{$\begin{array}{c}\text { No. of } \\
\text { patients (\%) }\end{array}$} & $\begin{array}{c}\text { Sex } \\
\text { (M/F) }\end{array}$ & \multicolumn{4}{c}{ Age distribution (rate, \%) } \\
\cline { 5 - 8 } & & $0-20 \mathrm{yr}$ & $21-40 \mathrm{yr}$ & $41-60 \mathrm{yr}$ & $>60 \mathrm{yr}$ \\
\hline MF & $115(22.2)$ & 1.1 & 3.5 & 36.5 & 41.7 & 18.3 \\
CD30 & $71(13.7)$ & 1.3 & 21.1 & 21.1 & 21.1 & 21.1 \\
SPTCL & $32(6.2)$ & 0.5 & 15.6 & 50.0 & 25.0 & 9.4 \\
PTL & $89(17.2)$ & 1.1 & 5.6 & 21.3 & 38.2 & 34.8 \\
NK/T & $62(12.0)$ & 1.5 & 11.3 & 16.1 & 29.0 & 43.5 \\
AITL & $13(2.5)$ & 5.5 & 0.0 & 0.0 & 23.1 & 76.9 \\
MZBL & $23(4.5)$ & 1.9 & 0.0 & 21.7 & 60.9 & 17.4 \\
DLBCL & $58(11.2)$ & 1.9 & 0.0 & 8.6 & 29.3 & 62.1 \\
BPDCN & $11(2.1)$ & 10.0 & 9.1 & 36.4 & 9.1 & 45.5 \\
T, LBL & $9(1.7)$ & 8.0 & 11.1 & 44.4 & 44.4 & 0.0 \\
B, LBL & $10(1.9)$ & 0.7 & 80.0 & 0.0 & 10.0 & 10.0 \\
Others & $24(4.7)$ & 2.0 & 4.2 & 0.0 & 33.3 & 62.5 \\
\hline
\end{tabular}

M, male; F, female; MF, mycosis fungoides; CD30, CD30+ T-cell lymphoproliferative disorder; SPTCL, subcutaneous panniculitis-like T cell lymphoma; PTL, peripheral T cell lymphoma; NKTT, NK/T cell lymphoma, nasal type; AITL, angioimmunoblastic T cell lymphoma; MZBL, marginal zone B-cell lymphoma; DLBCL, diffuse large B-cell lymphoma; BPDCN, blastic plasmacytoid dendritic cell neoplasm; LBL, lymphoblastic lymphoma.

cell lymphoma, and 5 cases were small/medium CD4-positive T-cell lymphoma. Of the 23 cases of primary cutaneous DLBCL, 6 were categorized as DLBCL, leg type.

Comparison of lymphoma subtype with sex and age revealed that BPDC neoplasm and T lymphoblastic lymphoma was predominantly associated with males, while CD30+ LPD, SPTCL, and lymphoblastic lymphoma occurred more often in younger individuals (Table 2). Likewise, comparison of lymphoma sub-
Table 3. Cutaneous lymphomas by histologic type and anatomic location

\begin{tabular}{lcccccr}
\hline & $\begin{array}{c}\text { No. of } \\
\text { patients } \\
\end{array}$ & \multicolumn{5}{c}{ Anatomic location (rate, \%) } \\
\cline { 3 - 7 } & $\begin{array}{c}\text { Head and } \\
\text { neck }\end{array}$ & Trunk & $\begin{array}{c}\text { Upper } \\
\text { limb }\end{array}$ & $\begin{array}{c}\text { Lower } \\
\text { limb }\end{array}$ & Multiple \\
\hline MF & $115(22.2)$ & 4.4 & 53.5 & 7.0 & 16.7 & 18.4 \\
CD30 & $71(13.7)$ & 8.6 & 28.6 & 28.6 & 27.1 & 7.1 \\
SPTCL & $32(6.2)$ & 3.1 & 53.1 & 12.5 & 12.5 & 18.8 \\
PTL & $89(17.2)$ & 15.9 & 37.5 & 13.6 & 26.1 & 6.8 \\
NKT & $62(12.0)$ & 6.5 & 30.6 & 17.7 & 38.7 & 6.5 \\
AITL & $13(2.5)$ & 30.8 & 46.2 & 23.1 & 0.0 & 0.0 \\
MZBL & $23(4.5)$ & 52.2 & 39.1 & 8.7 & 0.0 & 0.0 \\
DLBCL & $58(11.2)$ & 12.5 & 42.9 & 21.4 & 21.4 & 1.8 \\
BPDCN & $11(2.1)$ & 27.3 & 36.4 & 9.1 & 18.2 & 9.1 \\
T, LBL & $9(1.7)$ & 33.3 & 33.3 & 0.0 & 22.2 & 11.1 \\
B, LBL & $10(1.9)$ & 60.0 & 10.0 & 10.0 & 20.0 & 0.0 \\
Others & $24(4.7)$ & 12.5 & 45.8 & 8.3 & 29.2 & 4.2 \\
\hline
\end{tabular}

MF, mycosis fungoides; CD30, CD30+ T-cell lymphoproliferative disorder; SPTCL, subcutaneous panniculitis-like T cell lymphoma; PTL, peripheral T cell lymphoma; NKTT, NKTT cell lymphoma, nasal type; AITL, angioimmunoblastic T cell lymphoma; MZBL, marginal zone B-cell lymphoma; DLB$\mathrm{CL}$, diffuse large B-cell lymphoma; BPDCN, blastic plasmacytoid dendritic cell neoplasm; LBL, lymphoblastic lymphoma.

type with the involved anatomical site revealed that marginal zone B-cell lymphoma and B lymphoblastic lymphoma occurred more frequently in the head and neck (Table 3). In addition, the relative incidences of CD30+ LPD, NK/T cell lymphoma, SPTCL, and immature hematopoietic malignancies were higher while those of MF and B-cell lymphoma was lower when com- 
Table 4. Comparison of relative frequency of primary cutaneous lymphomas with reported incidences in Western countries

\begin{tabular}{lcc}
\hline & Western $(\%)^{5}$ & Present (\%) \\
\hline Mature T-cell lymphoma & 71.3 & 82.3 \\
MF & 38.3 & 30.4 \\
SS & 0.8 & 0.3 \\
CD30 & 10.2 & 16.9 \\
SPTCL & 0.6 & 8.2 \\
PTL & 20.8 & 18.0 \\
NKKT & 0.3 & 8.2 \\
AITL & 0.2 & 0.3 \\
Mature B-cell lymphoma & 28.5 & 13.0 \\
MZBL & 7.1 & 5.0 \\
FL & 8.5 & 1.1 \\
DLBCL & 11.4 & 6.1 \\
Immature hematologic malignancies & 0.3 & 4.0 \\
BPDCN & 0.2 & 2.7 \\
T, LBL & 0.0 & 0.5 \\
B, LBL & 0.1 & 0.8 \\
Others & 1.5 & 0.8 \\
\hline
\end{tabular}

MF, mycosis fungoides; SS, Sezary syndrome; CD30, CD30+ T-cell lymphoproliferative disorder; SPTCL, subcutaneous panniculitis-like T cell lymphoma; PTL, peripheral T cell lymphoma; NK/T, NK/T cell lymphoma, nasal type; AITL, angioimmunoblastic T cell lymphoma; MZBL, marginal zone Bcell lymphoma; FL, follicular lymphoma; DLBCL, diffuse large B-cell lymphoma; BPDCN, blastic plasmacytoid dendritic cell neoplasm; LBL, lymphoblastic lymphoma.

pared with one Western study (Table 4). ${ }^{5}$

\section{DISCUSSION}

In the present study we analyzed the incidence rate of Korean cutaneous lymphoma according to the new WHO-EORTC classification. To our knowledge, only a few nation-wide studies of Korean cutaneous lymphoma exist, all of which have several limitations. Specifically, one report focused on systemic lymphoma, ${ }^{1}$ reporting an incidence of lymphoma subtype very different from the results observed in this study, such as SPTCL being the most common cutaneous lymphoma. One possible explanation for this difference is that the previous report was based on the 2001 WHO classification and described a very limited set of lymphoma subtypes. Other reports have similar limitations. ${ }^{\text {? }}$

With respect to lymphoma subtype and sex, the results of our study were consistent with previous reports. ${ }^{5,8,9}$ Specifically, we observed a male predominance for the majority of lymphoma subtypes, especially BPDC neoplasm and T lymphoblastic lymphoma, and a female predominance for SPTCL. We also found that most patients were elderly, with a peak incidence around 60 years. As with other systemic lymphomas, aging and age-related effects such as chronic inflammation and diminished immune surveillance may contribute to lymphomagenesis. ${ }^{10}$

Our study included primary and secondary cutaneous lymphomas. However, the distinction between primary and secondary can be difficult because of incomplete clinical evaluation. Although undetermined cases accounted for only $0.8 \%$ of the study population, more problematic cases involving distinction between primary and secondary cutaneous lymphoma may exist. Secondary cutaneous involvement was relatively frequent in NK/T cell lymphoma, AITL, DLBCL, and lymphoblastic lymphoma. Furthermore, almost all of the patients in our study with AITL had systemic lymphoma, indicating secondary cutaneous involvement. AITL skin lesions are nonspecific subtle perivascular lymphoid cell infiltrates without atypia. Further, it is not clear whether such cutaneous manifestations are due to the tumor cell itself or secondary cytokine effects. Thus, immunohistochemical staining for follicular helper $\mathrm{T}$ cell markers such as PD-1 and CXCL13 may be helpful in making decisions.

In our study, $\mathrm{T}$ cell lymphoma comprised the majority (74.3\%) of cutaneous lymphomas, similar to studies conducted in Western countries. ${ }^{3,8,11}$ MF was the most common primary cutaneous lymphoma. MF displays various histologic appearances mimicking ALCL and PTCL if it transforms into large cells and progresses to the tumor stage from the plaque or patch stage. Before diagnosing ALCL or PTCL, a thorough patient clinical history and physical examination is needed. In the present study, among primary cutaneous lymphomas, the incidence of MF was $30.4 \%$, which was lower than reported for Western and Japanese studies. ${ }^{12,13}$ The reason for the relatively low incidence of MF in our study was probably due a result of not considering patient's clinical findings. Specifically, some PTCL cases may have belonged to the tumor stage of MF. Of the CD30+ LPD cases, there was disagreement with respect to LyP and ALCL between pathologists. Indeed, distinction of LyP from ALCL requires careful evaluation of clinical parameters and relative percentages of large atypical cells, which is a subjective parameter.

We found that there was a significant difference in incidence compared with Western countries, especially concerning NK/T cell lymphoma and SPTCL. These differences in incidences between countries were very likely the result of viral infection, especially Epstein-Barr virus (EBV) and human T-lymphotropic virus (HTLV). Indeed, EBV positivity is the most important finding in diagnosing NK/T cell lymphoma. Likewise, adult $\mathrm{T}$ cell leukemia/lymphoma is associated with HTLV infection and is endemic in several areas of the world, especially in Japan, ${ }^{14}$ but was rarely observed in the present study.

Two possibilities for the rarity of the incidence of the PTCL 
subtype can be suggested. First, this study selected cases from 2006. Compared with the 2001 WHO classification, primary cutaneous aggressive epidermotropic CD8-positive cytotoxic Tcell lymphoma, primary cutaneous $\gamma \delta$ T-cell lymphoma, and primary cutaneous small/medium CD4-positive T-cell lymphoma were included as a provisional entity in the new WHO-EORTC classification. Thus, the hemato/dermatopathologist would likely miss these cases. Second, it was difficult to differentiate the PTCL subtype from other cutaneous T-cell lymphomas. For example, primary cutaneous gamma-delta T-cell lymphoma was previously described as SPTCL with a gamma/delta phenotype that shares common morphologic and immunophenotype features. The most important immunophenotype marker for this type of disease is TCR $\delta$, for which detection has recently become available. As such, negativity for $\beta \mathrm{F} 1$ favors a diagnosis of gamma/delta T-cell lymphoma if TCR $\delta$ is not appropriate. ${ }^{15}$ More immunohistochemical staining results for $\beta \mathrm{F} 1$ and TCR $\delta$ are needed in cases of PTCL and SPTCL.

Subtypes of DLBCL were rarely observed in this study. Primary cutaneous DLBCL, leg type, presents on the leg, although other cutaneous sites may be involved. Furthermore, tumor cells of DLBCL, leg type, typically express Bcl2, Bcl6, and MUM1, although approximately $10 \%$ of cases do not express either Bcl2 or MUM1. ${ }^{16}$ By definition, DLBCL comprises all other cases other than those belonging to DLBCL, leg type, and to date only one marker, $\mathrm{Bcl} 6$, has been found to be useful in distinguishing between these two subtypes. One study investigated chromosomal aberrations in two subtypes of DLBCL and found similar aberrations irrespective of anatomical site, cell morphology, and $\mathrm{Bcl} 2$ expression. ${ }^{17}$ Among cases of cutaneous B-cell lymphoma, marginal zone B-cell lymphoma was relatively common while follicular lymphoma was rare; these findings were correlated with the results of previous lymphoma studies performed on Korean ${ }^{1}$ and Japanese populations. ${ }^{13}$

In addition PTCL and DLBCL subtypes, it is also important to pay attention to BPDC neoplasm, which is newly described and included in 4th edition of WHO classification. BPDC neoplasm is a subset of acute myeloid leukemia, related to precursor neoplasms, ${ }^{4}$ and is a clinically aggressive tumor derived from the precursors of plasmacytoid dendritic cells and characterized by a diffuse infiltration of blastic cells with irregular nuclei, fine chromatin and small nucleoli. BPDC neoplasm frequently involves the skin, and has also been described as blastic NK-cell lymphoma. ${ }^{2}$ Immunohistochemical staining for CD4, CD56, and $\mathrm{CD} 123$ is essential to confirm the diagnosis.

Regretfully, we were unable to evaluate patient outcome as- sociated with the new WHO-EORTC classification system. Thus, further studies concerning patient prognosis and efficacy of different treatment modalities are needed. However, our data were collected from many hospitals where hematopathologists involved in a large number of cases performed the reviews, and included all kinds of cutaneous lymphoma subtypes. In spite of limitations such as absence of comprehensive review of submitted cases, this study may represent the current status of cutaneous lymphoma subtypes in Korea according to new WHOEORTC classification.

In conclusion, compared with Western countries, mature Tcell lymphoma was found to be more frequent in Korean patients because of the frequent occurrence of CD $30+$ LPD, SPT$\mathrm{CL}$, and NK/T cell lymphoma. Conversely, there were fewer cases of B-cell lymphoma, especially follicular lymphomas. The new WHO-EORTC classification system is expected to contribute to uniform diagnosis in cutaneous lymphoma, and thus pathologists and dermatologists should recognize and adapt their diagnoses accordingly.

\section{Conflicts of Interest}

No potential conflict of interest relevant to this article was reported.

\section{Acknowledgments}

This research was supported by a grant from The Korean Society of Pathologists.

\section{REFERENCES}

1. Kim JM, Ko YH, Lee SS, et al. WHO classification of malignant lymphomas in Korea: report of the third nationwide study. Korean J Pathol 2011; 45: 254-60.

2. Leboit PE, Burg G, Weedon D, Sarasin A. Pathology and genetics of skin tumours. Lyon: IARC Press, 2006.

3. Willemze R, Jaffe ES, Burg G, et al. WHO-EORTC classification for cutaneous lymphomas. Blood 2005; 105: 3768-85.

4. Swerdlow SH, Campo E, Harris NL, et al. WHO classification of tumours of haematopoietic and lymphoid tissues. 4th ed. Lyon: IARC Press, 2008.

5. Bradford PT, Devesa SS, Anderson WF, Toro JR. Cutaneous lymphoma incidence patterns in the United States: a population-based study of 3884 cases. Blood 2009; 113: 5064-73.

6. Dores GM, Anderson WF, Devesa SS. Cutaneous lymphomas reported to the National Cancer Institute's surveillance, epidemiology, and end results program: applying the new WHO-European 
Organisation for Research and Treatment of Cancer classification system. J Clin Oncol 2005; 23: 7246-8.

7. Lee MW; Korean Dermatopathology Research Group. Characteristics of cutaneous lymphomas in Korea. Clin Exp Dermatol 2003; 28: 639-46.

8. Criscione VD, Weinstock MA. Incidence of cutaneous T-cell lymphoma in the United States, 1973-2002. Arch Dermatol 2007; 143: 854-9.

9. Morton LM, Turner JJ, Cerhan JR, et al. Proposed classification of lymphoid neoplasms for epidemiologic research from the Pathology Working Group of the International Lymphoma Epidemiology Consortium (InterLymph). Blood 2007; 110: 695-708.

10. Boren E, Gershwin ME. Inflamm-aging: autoimmunity, and the immune-risk phenotype. Autoimmun Rev 2004; 3: 401-6.

11. Riou-Gotta MO, Fournier E, Mermet I, et al. Primary cutaneous lymphomas: a population-based descriptive study of 71 consecutive cases diagnosed between 1980 and 2003. Leuk Lymphoma 2008; 49: 1537-44.

12. Fink-Puches R, Zenahlik P, Bäck B, Smolle J, Kerl H, Cerroni L. Primary cutaneous lymphomas: applicability of current classification schemes (European Organization for Research and Treatment of
Cancer, World Health Organization) based on clinicopathologic features observed in a large group of patients. Blood 2002; 99: 800-5.

13. Fujita A, Hamada T, Iwatsuki K. Retrospective analysis of 133 patients with cutaneous lymphomas from a single Japanese medical center between 1995 and 2008. J Dermatol 2011; 38: 524-30.

14. The World Health Organization classification of malignant lymphomas in Japan: incidence of recently recognized entities. Lymphoma Study Group of Japanese Pathologists. Pathol Int 2000; 50: 696-702.

15. Willemze R, Jansen PM, Cerroni L, et al. Subcutaneous panniculitislike T-cell lymphoma: definition, classification, and prognostic factors: an EORTC Cutaneous Lymphoma Group Study of 83 cases. Blood 2008; 111: 838-45.

16. Kodama K, Massone C, Chott A, Metze D, Kerl H, Cerroni L. Primary cutaneous large B-cell lymphomas: clinicopathologic features, classification, and prognostic factors in a large series of patients. Blood 2005; 106: 2491-7.

17. Wiesner T, Streubel B, Huber D, Kerl H, Chott A, Cerroni L. Genetic aberrations in primary cutaneous large B-cell lymphoma: a fluorescence in situ hybridization study of 25 cases. Am J Surg Pathol 2005; 29: 666-73. 